\title{
ETUDE PRÉLIMINAIRE DE L'IMPORTANCE DE LA CYSTICERCOSE PORCINE eN ABATtOIr À ANTANANArivo, MADAGascar *
}

\author{
Preliminary RePORt on CySticercosis in PIGS AT SLAUGHTERHOUSE \\ in Antananarivo, Madagascar
}

\author{
ESTUDIO PRELIMINAR DE LA FRECUENCIA DE LA CISTICERCÓSIS EN MATADEROS \\ en Antananario, Madagascar
}

\author{
H. Rasamoelina-Andriamanivo ${ }^{1 *}$ E.O. Rasamoelina ${ }^{1}$ V. Porphyre ${ }^{2}$
}

\begin{abstract}
Mots-clés : Porcin - Viande porcine - Cysticercose - Taenia solium - Surveillance - Abattoir - Madagascar.
\end{abstract}

Keywords: Swine - Pork - Cysticercosis - Taenia solium Monitoring - Slaughterhouse -Madagascar.

Palabras clave: Cerdo - Carne de cerdo - Cisticercósis Taenia solium - Vigilancia - Mataderos - Madagascar.

I a cysticercose est une maladie parasitaire largement répanLdue dans les pays en développement d'Amérique latine, $d^{\prime}$ Afrique et d'Asie. Cette maladie tropicale négligée représente une contrainte économique forte pour la filière porcine et un grave problème de santé publique à Madagascar. Plusieurs centaines de cas humains sont enregistrés chaque année dans les hôpitaux de la capitale malgache. Les éleveurs ruraux classent la cysticercose à la première place, devant les pestes porcines du fait de sa fréquence et de la perte économique qu'elle engendre. Cependant aucune donnée fiable sur l'importance réelle de la maladie chez le porc n'est disponible actuellement.

Dans cette étude, les objectifs ont été, d'une part, d'évaluer l'importance de la cysticercose porcine dans les abattoirs qui approvisionnent les marchés d'Antananarivo et, d'autre part, d'identifier ses facteurs de variation. Un suivi d'abattoirs a été mis en place à partir de mars 2013 pour une durée d'un an. Deux principaux lieux d'abattage à Antananarivo ont été choisis. Une inspection de tous les porcs abattus et la collecte des données sur les facteurs de variation (race, origine, sexe, date) ont été effectuées.

Après six mois de suivi, les résultats intermédiaires ont concerné I'abattoir d'Ankadindratombo. Sur 2904 porcs abattus, issus de 12 districts répartis dans toute l'île, la prévalence de la cysticercose a été de 3,9 p. 100. L'incidence mensuelle a été de 19 porcs infestés. Parmi les facteurs de variation, la prévalence a

1. Fofifa-Drzv, ministère de l'Enseignement supérieur et de la Recherche, Antananarivo, Madagascar.

2. Cirad, UMR Selmet, Saint-Pierre, Réunion, France.

* Auteur pour la correspondance

E-mail : harena23@gmail.com

$\$$ Ce texte est issu d'une communication orale présentée aux journées scientifiques QualiREG 2013. L'ensemble des résultats a ensuite fait l'objet d'une publication scientifique (Porphyre et coll., 2015) (1).

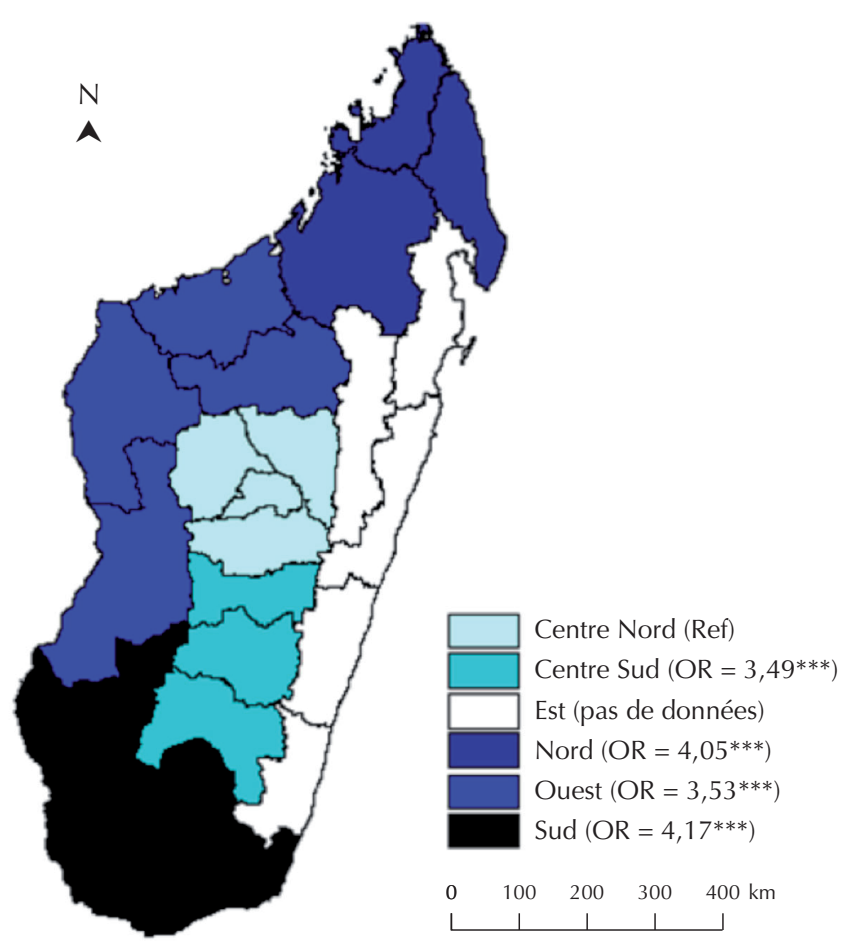

Figure 1 : cartographie du risque (odds ratio $[O R]$ ), selon l'origine des animaux, de détecter une carcasse infectée par Taenia solium pour un porc abattu dans I'abattoir d'Ankadindratombo, entre mars et août 2013, Antananarivo, Madagas$\operatorname{car}(* * * p<0,001)$.

varié selon les districts d'origine de 0 à 6 p. 100 (figure 1). Juillet a été le mois le moins à risque (odds ratio $=0,46 ; p=0,05$ ). La race locale a été plus à risque que les phénotypes exotiques (odds ratio $=2,40 ; p<0,001)$. Bien qu'en attente de données complètes, ces résultats confirment dès à présent l'importance de la maladie chez le porc.

\section{BIBLIOGRAPHIE}

1. PORPHYRE V., RASAMOELINA-ANDRIAMANIVO H., RAKOTOARIMANANA A., RASAMOELINA E.O., BENARD C., JAMBOU R., CARDINALE E., 2015. Spatio-temporal prevalence of porcine cysticercosis in Madagascar based on meat inspection. Parasites Vectors (in press)

Accepted 30 April 2015; Online publication June 2015 